\title{
Study on the Determination Model of Four Kinds of Tea Polyphenols in Fresh Tea Based on Visible and Near-infrared Spectroscopy
}

\section{Hailiang Zhang}

East China JiaoTong University

\section{Wentao Dong}

East China JiaoTong University

Wei Luo

East China JiaoTong University

\section{Baishao Zhan}

East China JiaoTong University

\section{Haihua Huang}

East China JiaoTong University

\section{Hong Chen}

East China JiaoTong University

Kai Min

East China JiaoTong University

Xuemei Liu ( $\nabla$ 12845024@qq.com )

East China JiaoTong University https://orcid.org/0000-0001-9542-8656

\section{Research}

Keywords: Visible and near-infrared spectroscopy, Tea polyphenols, Partial least squares, Multiple linear regression, Least squares support vector machine, Characteristic wavelengths

Posted Date: December 15th, 2020

DOl: https://doi.org/10.21203/rs.3.rs-127370/v1

License: (c) (i) This work is licensed under a Creative Commons Attribution 4.0 International License. Read Full License 
1

2

3 Hailiang Zhang ${ }^{1}$, Wentao Dong ${ }^{1}$, Wei Luo ${ }^{1}$, Baishao Zhan ${ }^{1}$, Haihua Huang ${ }^{1}$, Hong Chen $^{2}$, Kai

$4 \quad \mathrm{Min}^{3}$, Xuemei $\mathrm{Liu}^{3}, *$

5

6

7 330013, China

8

9

10

11

12

13

\section{Study on the determination model of four kinds of tea polyphenols} in fresh tea based on visible and near-infrared spectroscopy

${ }^{1}$ College of Electrical and Automation Engineering, East China Jiaotong University, Nanchang

${ }^{2}$ Rail Transit Technology Innovation Center, East China Jiaotong University, Nanchang 330013,

\section{China}

${ }^{3}$ College of Civil Engineering and Architecture, East China Jiaotong University, Nanchang 330013, China

\section{Abstract}

Background: For tea, tea polyphenols is an essential indicator to measure the quality of tea. In this paper, the content of four tea polyphenols in fresh tea was determined by visible and near-infrared spectroscopy combined with chemometrics.

Results: First, the spectrum data of three kinds of tea, Juhuachun (J), Zhenong25 (Z) and Yingshuang (Y) were collected. A total of 159 samples were collected, 106 of which were used for calibration and 53 for prediction. Then the content of tea polyphenols was determined by HPLC and the physicochemical value samples were established. Subsequently, the spectral data was 
preprocessed to eliminate noise interference, and a partial least squares (PLS) model was established to select the optimal preprocessing method. In order to improve the efficiency and accuracy of detection, Competitive adaptive reweighted sampling (CARS), Successive projections algorithm (SPA) and Random frog algorithm (RF) were used to extract characteristic wavelengths from the pretreatment spectrum. Based on characteristic wavelengths, PLS, multiple linear regression (MLR) linear models and least squares support vector machine (LS-SVM) nonlinear models were established to predict the content of four tea polyphenols. The performance of LS-SVM models is superior to that of PLS and MLR models. The $\mathrm{R}_{\mathrm{P}}{ }^{2}$ values of the four tea polyphenols LS-SVM models based on SPA and CARS were increased to 0.996, 0.991, 0.997, 0.988 and $0.997,0.991,0.997,0.984$, respectively. The $\mathrm{R}_{\mathrm{P}}{ }^{2}$ values of the four tea polyphenols LS-SVM models based on RF were also increased to $0.996,0.986,0.994$ and 0.977.

Conclusions: It can be found that the LS-SVM model based on SPA is the most suitable prediction model for the content of tea polyphenols. It has the least input variables and better performance. Therefore, visible and near-infrared spectroscopy can be used as an effective method to measure the content of tea polyphenols in fresh tea.

Keywords : Visible and near-infrared spectroscopy; Tea polyphenols; Partial least squares; Multiple linear regression; Least squares support vector machine; Characteristic wavelengths;

\section{Introduction}

Green tea is one of the three drinks with the largest consumption in the world because of its unique taste, high nutritional value and health care effect on human body. Tea polyphenols are also 
the most important functional substance in tea. Its main components not only affect the taste of tea, but also have a remarkable influence on the nutritional value of tea. The content difference of tea polyphenols in tea leaves has important influence on the quality of tea leaves.

Tea polyphenols are the general term for polyphenol compounds in tea and its main component is catechin. There are 9 kinds of catechin monomers, of which 4 monomers, EGC (epigallocatechin), EGCG (epigallocatechin gallate), EC (epicatechin) and ECG (epicatechin gallate) content is higher than other monomers [1]. Studies have shown that tea polyphenols, as an antioxidant, have a preventive effect on cancer and various cardiovascular diseases [2]. It can improve the immunity of human body and have a good health care effect on human body, which is an important symbol to measure the quality of tea [3].

At present, the detection of tea polyphenols and other chemical components in tea mainly adopts high performance liquid chromatography (HPLC) [4]. This method has some disadvantages such as longer analysis time, more complicated operation and higher requirement on instrument and equipment. The non-destructive spectroscopy technology can provide sample information without damaging the sample. Therefore, in recent years, the spectral detection technology has been studied deeply [5] and the spectral based tea quality detection technology has also been developing continuously. Zhang et al. [6] used visible and near-infrared spectroscopy to rapidly detect the water content in tea leaves. Jin [7] achieved rapid quantitative detection of physiological and biochemical indicators of tea under heavy metal stress based on hyperspectral imaging technology. Dutta et al. [8] combined remote sensing technology and near-infrared spectroscopy to identify the quality of black tea and green tea. Chen et al. [9] used surface-enhanced Raman spectroscopy to analyze the quality of tea. 
Near-infrared spectroscopy technology has been widely used in the detection of tea chemical components in recent years because of its fast, simple and portable characteristics. Bai et al. [10] established a detection model for tea polyphenols, moisture and caffeine content in tea powder based on near-infrared spectroscopy technology. Liu Ze et al. [11] rapidly determined tea polyphenols and other compounds in Pu'er tea by combining near-infrared spectroscopy and weighted partial least square method. Zhang [12] used near-infrared spectroscopy to detect the content of tea polyphenols in different leaf positions.

In this article, three tea varieties were used as samples. After preprocessing the spectral data by different methods, established a partial least squares regression model. Then, successive projections algorithm (SPA), competitive adaptive reweighted sampling (CARS) and random frog (RF) were used to extract the characteristic wavelengths. Based on the characteristic wavelengths, partial least squares regression model (PLS), multiple linear regression model (MLR) and least square support vector machine model (LS-SVM) were established respectively. And through the determination coefficient $\left(\mathrm{R}^{2}\right)$ and root mean square error (RMSE) to evaluate the predictive ability of the model.

The objectives of this study were: (1) to explore the feasibility of visible and near-infrared spectroscopy in predicting tea polyphenols content; (2) to evaluate the performance of the three kinds of method to extract characteristic wavelengths; (3) to establish a high efficiency and high accuracy predictive model to detect the content of tea polyphenols in tea.

\section{Materials and methods}

\subsection{Sample preparation}


Three tea samples were used in this experiment, Juhuachun (J), Zhenong25 (Z) and Yingshuang (Y), which were picked at the Huajiachi Campus of Zhejiang University (120.206 E, $30.274 \mathrm{~N}$ ). A total of 159 tea samples were obtained for the experiment, and all the samples were divided into calibration set and prediction set according to 2:1. There were 106 samples in the calibration set and 53 samples in the prediction set.

\subsection{Spectra acquisition}

The Vis-NIR spectra were collected by using a near infrared spectrometer (XDS, Foss Pacific Pty Ltd, Denmark) in this experiment. Spectral resolution of $2 \mathrm{~nm}$ over the range of 400-2498 nm, and it contains 1050 bands. To simulate the state of the tea leaves just picked, no tea samples were processed. In order to improve the signal to noise ratio, each tea sample was scanned three times and the average data of the three spectra was used for the next analysis. The spectral collection and analysis were conducted by the ISIscan 1.50 (Infrasoft International LLC, State College, PA, USA).

\subsection{HPLC measurement}

After collecting the spectra, the tea samples were frozen in a freeze-dryer (LABCONCO, USA) for 24 hours. Then, the sample was ground into a powder with a grinder (FW100, Taisite Instrument Co., Ltd., Tianjin, China) and sieved through a 60-mesh sieve to obtain a sample of tea powder. Subsequently, $0.1 \mathrm{~g}$ of each tea powder sample was weighed and $25 \mathrm{~mL}$ water was added to stir evenly. The tea powder was heated in a water bath at $85^{\circ} \mathrm{C}$ for $20 \mathrm{~min}$. The supernatant was filtered by a $0.22 \mu \mathrm{m}$ membrane filter and then analyzed by HPLC [13]. The whole experiment was conducted at room temperature and in the dark to avoid the decomposition of tea polyphenols. 
UV-Visible detector (wavelength range: 190-600 nm) was used. Mobile phase were: (A) acetic acid/acetonitrile/distilled water $(0.5: 3: 96.5)$ and (B) acetic acid/acetonitrile/distilled water (0.5:30:69.5). The flow rate was $1.0 \mathrm{~mL} / \mathrm{min}$ and the injection volume was $10 \mu \mathrm{L}$. The UV detection wavelength was $280 \mathrm{~nm}$ and the column temperature was maintained at $35^{\circ} \mathrm{C}$.

\subsection{Spectral preprocessing} through near-infrared spectroscopy may also include noise pollution and baseline drift caused by some equipment or the external environment. It will reduce the accuracy of the calibration model. 10.1.

\subsection{Extraction of characteristic wavelengths}

NIR spectroscopy usually contains thousands of variables, of which quite a few variables have a collinearity relationship and some variables contain useless information. Therefore, using full spectral data for modeling will not only increase the calculation time of the model and reduce the detection efficiency, but also has a certain impact on the predictive ability of the model. To address this problem, it is necessary to extract characteristic wavelengths from the full spectrum 
127

data to reduce the dimension of the NIR spectrum [14]. The CARS, SPA and RF were used to extract characteristic wavelengths in this paper.

\subsubsection{Competitive adaptive reweighted sampling}

CARS is a common method for selecting spectral variables. By combining exponentially decreasing function (EDF) with adaptive reweighted sampling (ARS), the wavelength points with large absolute regression coefficient in the PLS model are selected and the wavelength points with small weight are eliminated. Then, the lowest the root mean square error (RMSE) subset is selected as the characteristic wavelength set through cross-validation [15]. The running process of CARS mainly includes the following four steps: (1) Sample the model by Monte Carlo method; (2) Remove variables by exponentially decreasing function; (3) Remove variables further by adaptive reweighted sampling; (4) Calculate the RMSEV value of the variable subset, and selecting the subset with the smallest RMSEV as the characteristic wavelength set [16].

\subsubsection{Successive projections algorithm}

SPA is mainly used to solve collinearity problems in multiple linear regression problems. In recent years, it has been widely used. SPA is a forward cycle selection method, which begins with a wavelength, successive cycle forward calculation. Each time the projection of the selected wavelength on the unselected wavelength is calculated, the wavelength with the largest projection distance is introduced into the characteristic wavelength set. Finally, a multiple linear regression model is established for each selected wavelength to obtain RMSE of the validation set. The smallest RMSE the characteristic wavelength subset is the optimal set [17]. SPA can select the wavelength with minimum collinearity from all spectral data to reduce the number of wavelengths 
148

149

150

151

152

153

154

155

156

required for model establishment. Thus, it can improve detection efficiency. SPA mainly includes the following three steps: (1) Calculate the projection of the selected wavelength on the unselected wavelength through the algorithm, and obtain the candidate subset with the minimum collinearity variable; (2) Calculate the RMSEV of the validation set using multiple linear regression; (3) Select the subset corresponding to the minimum RMSEV and remove the irrelevant variables [18].

\subsubsection{Random frog algorithm}

Random frog algorithm is an algorithm proposed based on inspiring group optimization to solve combinatorial optimization problems. It constructs a Markov Chain in the model space to calculate the probability of each variable being selected, and selects the characteristic wavelength according to the probability of being selected. RF has high detection efficiency and excellent global search capability. The main steps of RF are divided into the following 3 steps: (1) Initialize the variable subset $\boldsymbol{V}_{\boldsymbol{0}}$ containing variable $\boldsymbol{Q}$, the number of iterations $\boldsymbol{N}$ and other parameters; (2) Propose a candidate subset $V^{*}$ containing variable $Q^{*}$ based on $V_{\boldsymbol{0}}$, take $V^{*}$ with a certain probability as $\boldsymbol{V}_{\boldsymbol{l}}$ and replace $\boldsymbol{V}_{\boldsymbol{0}}$ with $\boldsymbol{V}_{\boldsymbol{l}}$. Then, perform a new round of iterations until $\boldsymbol{N}$ iterations are performed; (3) Calculate the probability of each wavelength being selected. The greater the probability, the more important the wavelength [19]. Thus, the characteristic wavelength is selected.

\subsection{Model establishment and evaluation}

In this paper, PLS and MLR were used to establish a linear model and LS-SVM was used to establish a nonlinear model to predict four kinds of tea polyphenol monomers.

PLS is the most widely used modeling method in spectral analysis due to its advantages of 
169

170

171

172

173

174

convenience, stability, accuracy, and wide applicability [20]. In PLS analysis, the information in the spectral matrix and the concentration matrix can be considered at the same time. Thereby improving the predictive ability of the model and having the ability to process a large amount of spectral data [21]. Therefore, PLS was used to model the full spectrum data and characteristic wavelengths in this paper. And through the performance of the PLS model to evaluate the effect of the pretreatment method.

MLR is a method used to analyze the relationship between multiple independent variables and a single dependent variable. It is often used to deal with linear problems in models [22]. MLR is only applicable to data with a sample number more than the spectral number, so MLR is often used to establish a calibration model based on the characteristic wavelength.

LS-SVM is an improved algorithm based on support vector machine. It replaces the more complicated quadratic programming method in the original SVM by solving a set of linear equations to increase the speed of calculation and reduce the complexity [23]. The input variables with rich information content can improve the operational efficiency of LS-SVM, so LS-SVM can deal with both linear and nonlinear problems [24].

After the model was established, the predictive performance of the model was evaluated by $\mathrm{R}^{2}$, RMSE and residual predictive deviation (RPD), respectively [25]. The calculation formula of these indicators is as follows:

$$
R^{2}=1-\sum_{i=1}^{n}\left(y_{i}-\hat{y}_{i}\right)^{2} / \sum_{\mathrm{i}=1}^{\mathrm{n}}\left(y_{i}-\bar{y}_{i}\right)^{2}
$$




$$
R P D=\frac{1}{\sqrt{1-R_{P}^{2}}}
$$

Where $n$ is the number of samples, $y_{i}$ is the actual chemical value of the $i$ th sample, $\hat{y}_{i}$

191 is the predicted value of the $i$ th sample, and $\bar{y}_{i}$ is the average of the actual chemical values of all

192 samples. This study was conducted in Unscrambler10.1 (CAMO PRECESS AS, Oslo, Norway) and Matlab2010a (The Math Works, Natick, USA) .

\section{Results and discussion}

\subsection{Vis-NIR spectroscopy analysis}

NIR spectroscopy is based on the stretching vibration and combined frequency between molecules to absorb the spectrum. Because the spectral absorption characteristics of different groups are significantly different, the near-infrared spectrum can reflect the molecular structure and composition information of the sample. The Vis-NIR spectroscopy of tea samples within the range of $400 \mathrm{~nm}-2500 \mathrm{~nm}$ are shown in Figure. 1. The visible region is $400-800 \mathrm{~nm}$, and the near-infrared region is $800-2500 \mathrm{~nm}$.

There were two absorption peaks in the visible region, located at $480 \mathrm{~nm}$ and $670 \mathrm{~nm}$, which were mainly caused by the absorption of O-bonds and $\mathrm{O}-\mathrm{H}$ bonds [26]. There were 7 main absorption peaks in the near-infrared region, and the relatively flat absorption peak at $1200 \mathrm{~nm}$ was mainly caused by the $\mathrm{C}-\mathrm{H}$ bond stretching of $\mathrm{CH}_{2}$. The absorption peak around $1470 \mathrm{~nm}$ was generated by the $\mathrm{O}-\mathrm{H}$ and $\mathrm{C}-\mathrm{H}$ bonds. The absorption peak near $1735 \mathrm{~nm}$ was generated by the vibration of the $\mathrm{C}-\mathrm{H}$ bond of $\mathrm{CH}_{3}$. While the strong absorption peak at $1930 \mathrm{~nm}$ was caused by the combined transformation of $\mathrm{O}-\mathrm{H}$ bond and $\mathrm{HOH}$ [27]. The absorption peak at 2140nm was 

generated by the combination of $\mathrm{N}-\mathrm{H}$ and $\mathrm{C}=\mathrm{O}$ bond vibration. The weak absorption peaks at $2310 \mathrm{~nm}$ and $2350 \mathrm{~nm}$ were generated by $\mathrm{C}-\mathrm{H}$ bond bending and $\mathrm{C}-\mathrm{H}$ bond stretching, respectively [28].

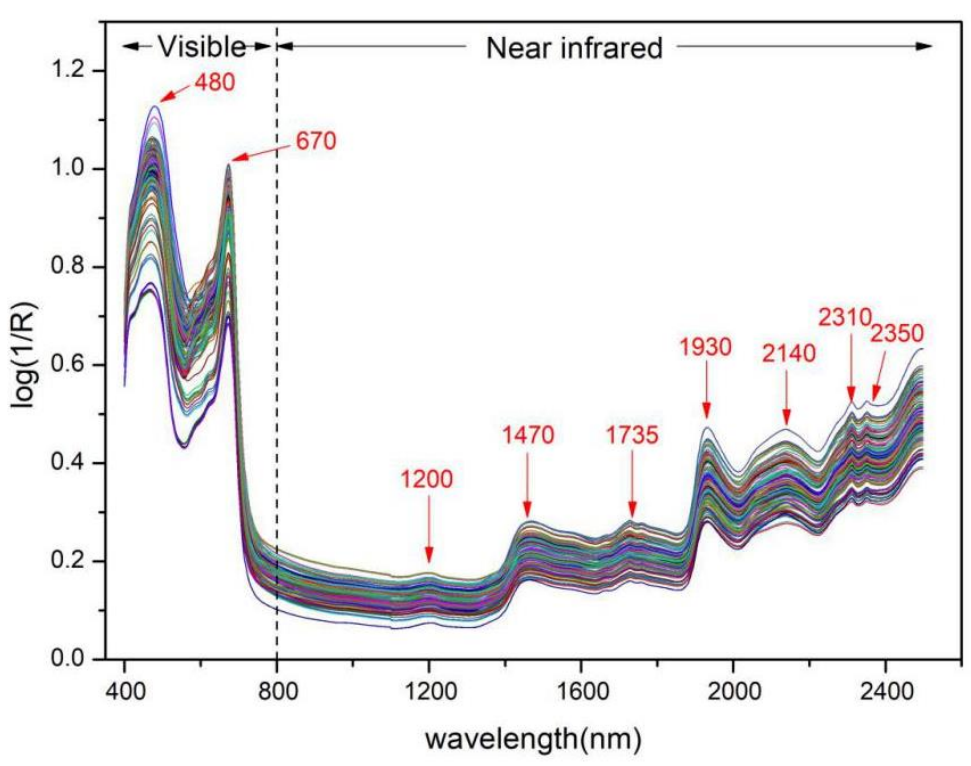

Fig. 1 Visible and near-infrared spectroscopy of tea samples

\subsection{Comparison of different spectral preprocessing methods}

The spectral preprocessing can eliminate the undesirable factors such as noise interference and baseline drift during the experiment. Three different pretreatment methods, SG smoothing, SNV and baseline, were used to process the original spectral data in this paper. And the PLS model was established with the processed data to evaluate the advantages and disadvantages of the preprocessing method according to the model capability. The PLS models of 4 kinds of tea polyphenols based on 3 preprocessing methods and original spectra are shown in Table 1.

As can be seen from the table that for the EGC monomer, the model after SNV treatment has the best effect, which can slightly improve the performance of the model. As far as EGCG is 
225 Similar to EGCG, the ECG model based on raw spectral data is superior to the model built after

226 data preprocessing. From what has been discussed above, in the subsequent research, EGC and EC

227 monomers were pretreated by SNV, and raw spectral data were used for modeling for EGCG and 228 ECG.

Table 1 The PLS model processed by different preprocessing methods

\begin{tabular}{|c|c|c|c|c|c|c|c|c|}
\hline Constituent & Pretreatment & RMSEC & $\mathrm{R}_{\mathrm{C}}^{2}$ & RMSEV & $\mathrm{Rv}_{\mathrm{v}}^{2}$ & RMSEP & $\mathrm{R}_{\mathrm{P}}^{2}$ & RPD \\
\hline \multirow{4}{*}{ EGC } & Raw & 6.974 & 0.932 & 7.889 & 0.914 & 6.882 & 0.933 & 3.863 \\
\hline & SNV & 6.580 & 0.940 & 7.761 & 0.917 & 6.592 & 0.939 & 4.048 \\
\hline & Baseline & 6.863 & 0.933 & 7.859 & 0.915 & 7.120 & 0.928 & 3.726 \\
\hline & SG & 6.974 & 0.931 & 7.889 & 0.914 & 6.882 & 0.933 & 3.863 \\
\hline \multirow{4}{*}{ EGCG } & Raw & 9.887 & 0.953 & 11.163 & 0.941 & 9.687 & 0.955 & 4.714 \\
\hline & SNV & 11.935 & 0.931 & 14.413 & 0.902 & 11.658 & 0.935 & 3.922 \\
\hline & Baseline & 11.681 & 0.934 & 13.239 & 0.917 & 11.710 & 0.934 & 3.892 \\
\hline & SG & 9.888 & 0.953 & 11.164 & 0.941 & 9.688 & 0.954 & 4.662 \\
\hline \multirow{4}{*}{$\mathrm{EC}$} & Raw & 2.461 & 0.915 & 2.934 & 0.881 & 2.480 & 0.913 & 3.390 \\
\hline & SNV & 2.460 & 0.916 & 3.033 & 0.873 & 2.450 & 0.916 & 3.450 \\
\hline & Baseline & 2.432 & 0.917 & 2.863 & 0.887 & 2.473 & 0.914 & 3.409 \\
\hline & SG & 2.461 & 0.915 & 2.935 & 0.881 & 2.481 & 0.913 & 3.390 \\
\hline \multirow{4}{*}{ ECG } & Raw & 5.460 & 0.893 & 6.221 & 0.864 & 5.467 & 0.892 & 3.042 \\
\hline & SNV & 5.515 & 0.891 & 6.161 & 0.866 & 5.545 & 0.889 & 3.001 \\
\hline & Baseline & 5.619 & 0.887 & 6.366 & 0.857 & 5.550 & 0.889 & 3.001 \\
\hline & SG & 5.462 & 0.893 & 6.222 & 0.864 & 5.468 & 0.891 & 3.028 \\
\hline
\end{tabular}


233 Therefore, in order to solve this problem, CARS, SPA and RF were used in this study to extract

234 characteristic wavelengths from the pretreatment spectrum data [29].
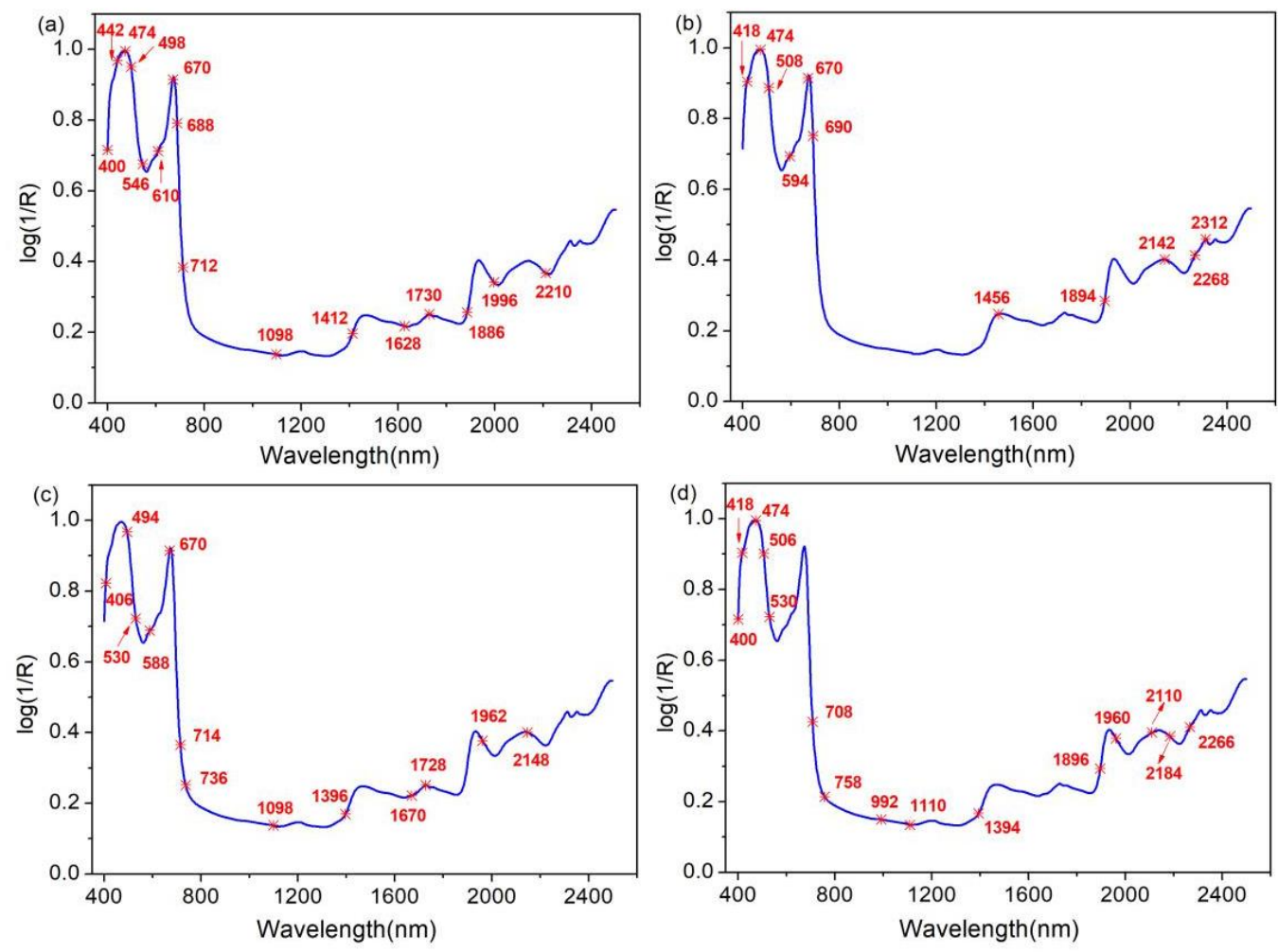
Fig. 2 The characteristic wavelength is extracted from the optimal pretreatment spectrum

(c)EC

(d)ECG

\subsection{The establishment of linear model}

After the characteristic wavelength extraction, the number of wavelength is greatly reduced and the detection efficiency is improved. Subsequently, the characteristic wavelength extracted based on CARS、SPA and RF are used to establish PLS and MLR models, and compared with the PLS model established at the full wavelength to evaluate the performance of the model. The modeling results of the PLS model and MLR model established with different wavelengths are shown in Table 2 and Table 3.

As can be seen from Table 2, the PLS model based on full wavelength is the best for EGC. The performance of models established based on CARS, SPA and RF extracted characteristic wavelengths have decreased, which may be caused by excluding some useful information when extracting characteristic wavelengths. But $\mathrm{R}_{\mathrm{P}}{ }^{2}$ still has 0.914 and 0.923 , the performance of the model is still acceptable. The EGCG model is similar to EGC. The model based on the full wavelength has the best effect, but the model based on CARS is close to the full wavelength model. As far as EC is concerned, the model based on CARS has the best performance and $\mathrm{R}_{\mathrm{P}}{ }^{2}$ is increased to 0.928 . For ECG, the model based on SPA has the best performance. The $\mathrm{R}_{\mathrm{P}}{ }^{2}$ is 0.898 , which is close to 0.9, and the effect is acceptable. It can be seen from Table 3 that, for all tea polyphenol monomers, the MLR model based on the characteristic wavelength extracted by CARS has the best performance. As far as EGCG is concerned, the performance of the MLR model based on RF is better than that of the model based on SPA. For EGC, EC and ECG, the 

methods. The $\mathrm{R}_{\mathrm{P}}{ }^{2}$ value of the model based on the characteristic wavelength is above 0.9 or close to 0.9 , and the effect is within the acceptable range. Moreover, it simplifies the input wavelength

272 and improves the detection efficiency.

Table 2 PLS models of four tea polyphenols based on different wavelengths

\begin{tabular}{|c|c|c|c|c|c|c|c|c|}
\hline Constituent & Pretreatment & Num & RMSEC & $\mathrm{R}_{\mathrm{C}}^{2}$ & RMSEV & $\mathrm{Rv}_{\mathrm{v}}^{2}$ & RMSEP & $\mathrm{R}_{\mathrm{P}}^{2}$ \\
\hline \multirow{4}{*}{ EGC } & \multirow{4}{*}{ SNV } & 1050 & 6.580 & 0.940 & 7.761 & 0.917 & 6.592 & 0.939 \\
\hline & & 16 & 7.296 & 0.925 & 8.323 & 0.904 & 7.400 & 0.923 \\
\hline & & 49 & 7.213 & 0.927 & 8.300 & 0.905 & 7.800 & 0.914 \\
\hline & & 20 & 7.797 & 0.914 & 9.227 & 0.882 & 8.068 & 0.908 \\
\hline \multirow{4}{*}{ EGCG } & \multirow{4}{*}{ Raw } & 1050 & 9.887 & 0.953 & 11.163 & 0.941 & 9.687 & 0.955 \\
\hline & & 11 & 11.078 & 0.941 & 12.410 & 0.927 & 10.909 & 0.943 \\
\hline & & 38 & 10.150 & 0.950 & 11.757 & 0.935 & 9.702 & 0.954 \\
\hline & & 36 & 11.678 & 0.934 & 12.933 & 0.921 & 10.753 & 0.944 \\
\hline \multirow{4}{*}{$\mathrm{EC}$} & \multirow{4}{*}{ SNV } & 1050 & 2.460 & 0.916 & 3.033 & 0.873 & 2.450 & 0.916 \\
\hline & & 13 & 2.952 & 0.878 & 3.418 & 0.839 & 2.965 & 0.877 \\
\hline & & 49 & 2.107 & 0.937 & 2.444 & 0.918 & 2.269 & 0.928 \\
\hline & & 15 & 2.094 & 0.938 & 2.334 & 0.925 & 2.059 & 0.940 \\
\hline \multirow{2}{*}{ ECG } & \multirow{2}{*}{ Raw } & 1050 & 5.460 & 0.893 & 6.221 & 0.864 & 5.467 & 0.892 \\
\hline & & 15 & 5.239 & 0.901 & 5.845 & 0.880 & 5.325 & 0.898 \\
\hline
\end{tabular}




$\begin{array}{lllllll}50 & 5.682 & 0.884 & 6.459 & 0.853 & 5.727 & 0.882 \\ 23 & 5.460 & 0.893 & 5.976 & 0.875 & 5.670 & 0.884\end{array}$

274

275

\begin{tabular}{|c|c|c|c|c|c|c|}
\hline Constituent & Pretreatment & Num & RMSEC & $\mathrm{R}_{\mathrm{C}}^{2}$ & RMSEP & $\mathrm{R}_{\mathrm{P}}^{2}$ \\
\hline \multirow{3}{*}{ EGC } & \multirow{3}{*}{ SNV } & 16 & 5.004 & 0.979 & 5.349 & 0.964 \\
\hline & & 49 & 2.367 & 0.992 & 4.668 & 0.984 \\
\hline & & 20 & 5.402 & 0.977 & 5.695 & 0.959 \\
\hline \multirow{3}{*}{ EGCG } & \multirow{4}{*}{ Raw } & 11 & 9.687 & 0.977 & 10.145 & 0.950 \\
\hline & & 38 & 5.612 & 0.990 & 6.368 & 0.984 \\
\hline & & 36 & 7.201 & 0.980 & 9.032 & 0.975 \\
\hline \multirow{3}{*}{$\mathrm{EC}$} & & 13 & 1.725 & 0.979 & 1.812 & 0.954 \\
\hline & \multirow[t]{2}{*}{ SNV } & 49 & 0.874 & 0.989 & 1.476 & 0.984 \\
\hline & & 15 & 1.664 & 0.980 & 1.772 & 0.956 \\
\hline \multirow{3}{*}{ ECG } & \multirow{3}{*}{ Raw } & 15 & 4.282 & 0.961 & 4.630 & 0.934 \\
\hline & & 50 & 2.494 & 0.977 & 3.872 & 0.973 \\
\hline & & 23 & 4.196 & 0.968 & 4.089 & 0.940 \\
\hline
\end{tabular}

276

277

278

279

280

281

282 and RF is used as the input of the model. The performance of the model is shown in Table 4. The

Table 3 MLR models of four tea polyphenols based on different wavelengths

\subsection{The establishment of nonlinear model}

As PLS and MLR are both linear calibration methods, to further improve the accuracy of the model, a nonlinear modeling method is used for research. This article uses the LS-SVM algorithm to build the model. LS-SVM is an improved algorithm based on the SVM algorithm, which can effectively deal with linear and nonlinear problems in multivariate analysis, and is a common nonlinear modeling method. In this paper, the characteristic wavelength extracted by CARS, SPA scatter plots of model predicted values and measured values are shown in Figure. 3, Figure. 4 and 
Figure. 5.

By comparing Table 2, Table 3 and Table 4, it can be seen that compared with the PLS and MLR models, the predictive ability of the LS-SVM model has been greatly improved. The $\mathrm{R}_{\mathrm{P}}{ }^{2}$ values of the four tea polyphenols models based on SPA and CARS were increased to 0.996, 0.991, 0.997, 0.988 and $0.997,0.991,0.997,0.984$, respectively. The $\mathrm{R}_{\mathrm{P}}{ }^{2}$ values of the four tea polyphenols models based on RF were also increased to $0.996,0.986,0.994$ and 0.977 . This indicates that there is a nonlinear relationship between the spectral information and the content of tea polyphenol. The LS-SVM model can process linear and nonlinear relationships at the same time, so the performance of the model is relatively excellent. In summary, the LS-SVM model based on the characteristic wavelength extracted by SPA, CARS and RF has achieved good prediction effect. Among them, the LS-SVM model based on SPA is most suitable for detecting the content of tea polyphenols. This model has the least number of input wavelengths, improves the detection efficiency, and achieves relatively excellent results. It has high scientific significance for realizing rapid non-destructive testing.

Table 4 LS-SVM models of four tea polyphenols based on characteristic wavelengths

\begin{tabular}{|c|c|c|c|c|c|c|}
\hline Constituent & Pretreatment & Num & RMSEC & $\mathrm{R}_{\mathrm{C}}^{2}$ & RMSEP & $\mathrm{R}_{\mathrm{P}}^{2}$ \\
\hline \multirow{3}{*}{ EGC } & \multirow{3}{*}{ SNV } & 16 & 0.015 & 0.999 & 1.586 & 0.996 \\
\hline & & 49 & 0.660 & 0.999 & 1.386 & 0.997 \\
\hline & & 20 & 0.876 & 0.998 & 1.508 & 0.996 \\
\hline \multirow{3}{*}{ EGCG } & \multirow{3}{*}{ Raw } & 11 & 2.718 & 0.996 & 4.230 & 0.991 \\
\hline & & & & & & \\
\hline & & 38 & 2.598 & 0.996 & 4.132 & 0.991 \\
\hline
\end{tabular}




\begin{tabular}{|c|c|c|c|c|c|c|}
\hline & & 36 & 3.891 & 0.992 & 5.365 & 0.986 \\
\hline \multirow{3}{*}{$\mathrm{EC}$} & \multirow{3}{*}{ SNV } & 13 & 0.113 & 0.999 & 0.410 & 0.997 \\
\hline & & 49 & 0.134 & 0.999 & 0.395 & 0.997 \\
\hline & & 15 & 0.306 & 0.998 & 0.631 & 0.994 \\
\hline \multirow{3}{*}{ ECG } & \multirow{3}{*}{ Raw } & 15 & 1.094 & 0.995 & 1.799 & 0.988 \\
\hline & & 50 & 1.451 & 0.992 & 2.070 & 0.984 \\
\hline & & 23 & 1.862 & 0.987 & 2.517 & 0.977 \\
\hline
\end{tabular}
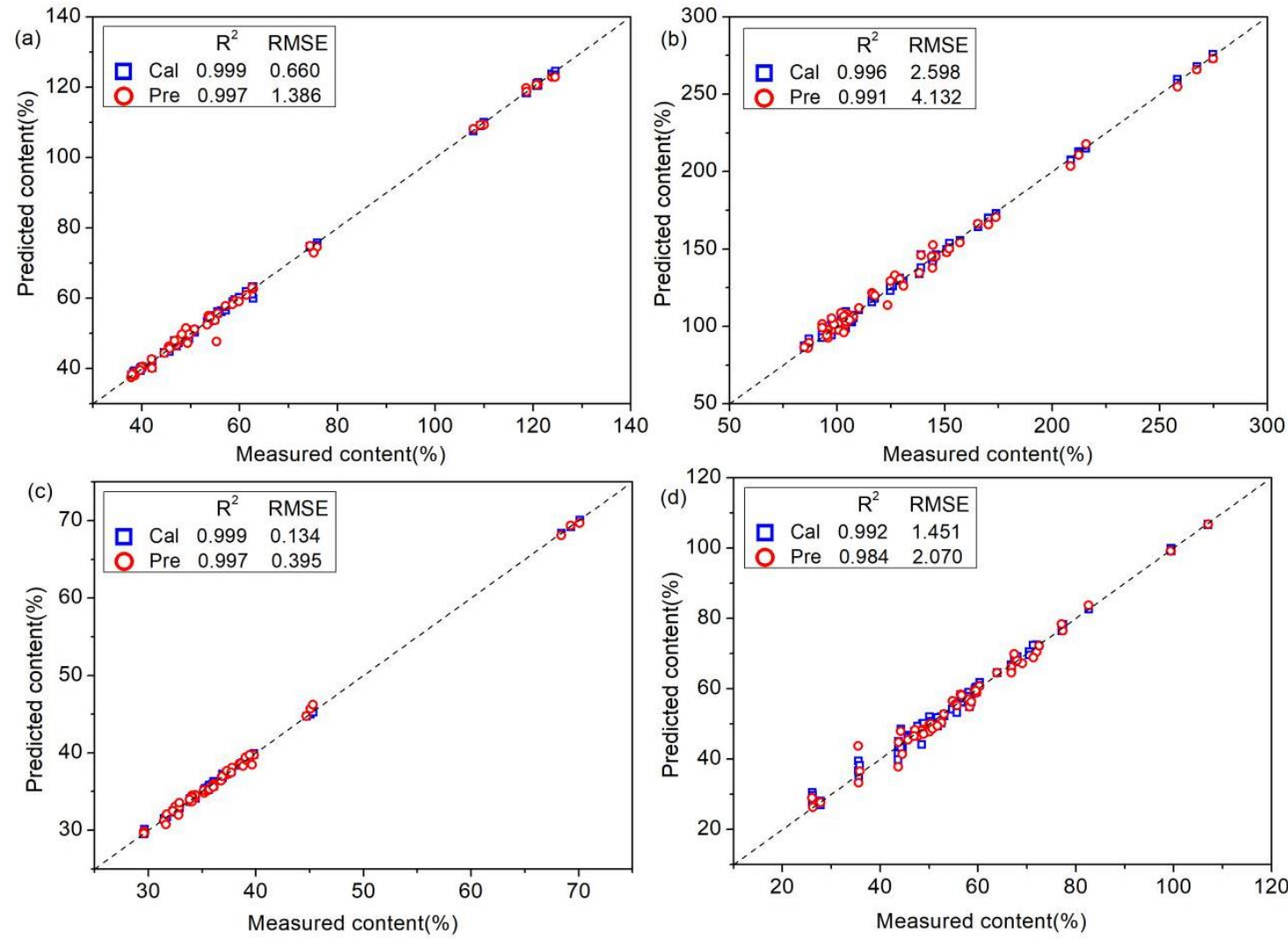

Fig. 3 Scatter plot of four tea polyphenol LS-SVM models based on CARS, (a)EGC 

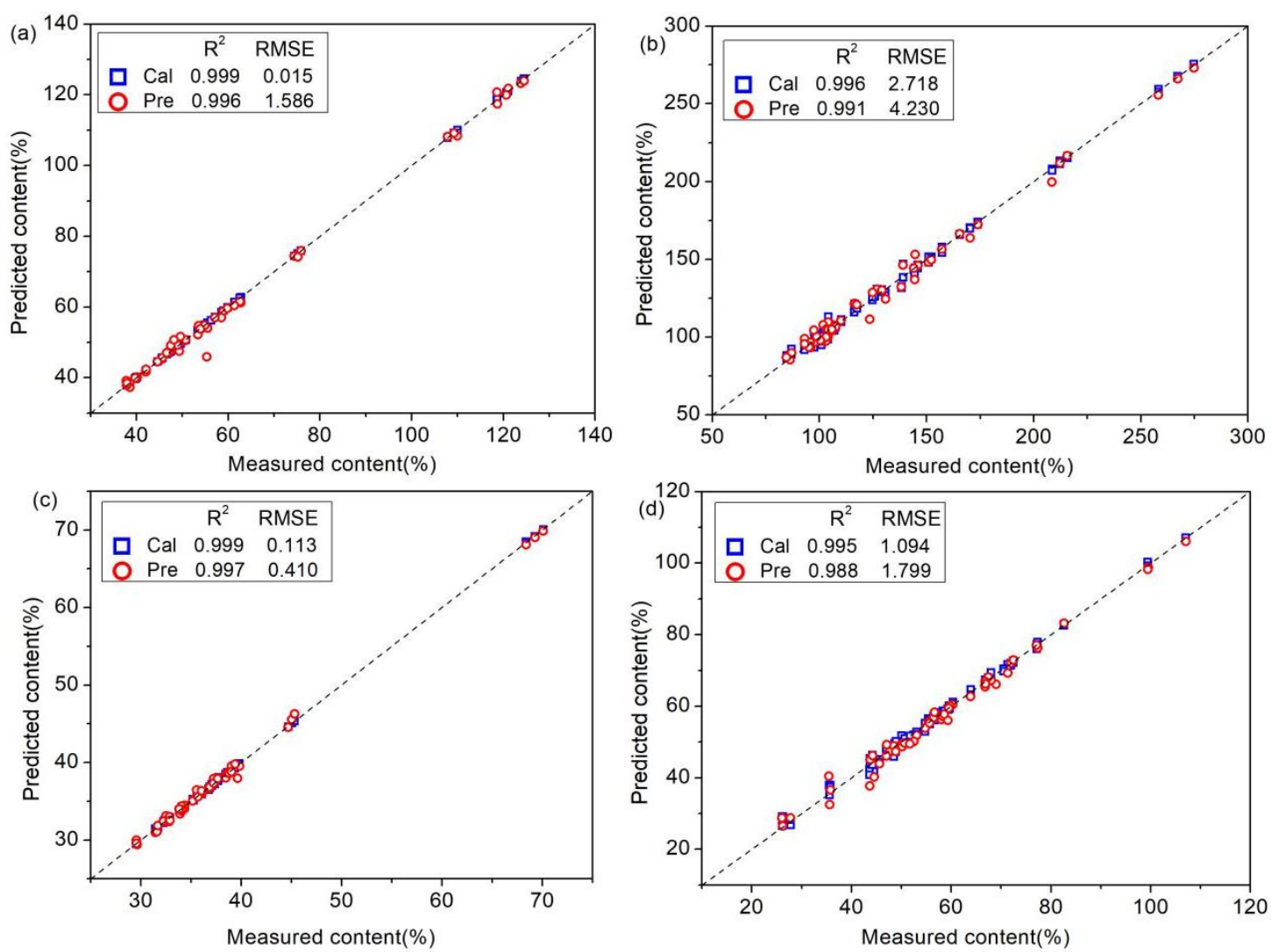

Fig. 4 Scatter plot of four tea polyphenol LS-SVM models based on SPA, (a)EGC (b)EGCG

(c)EC (d)ECG 

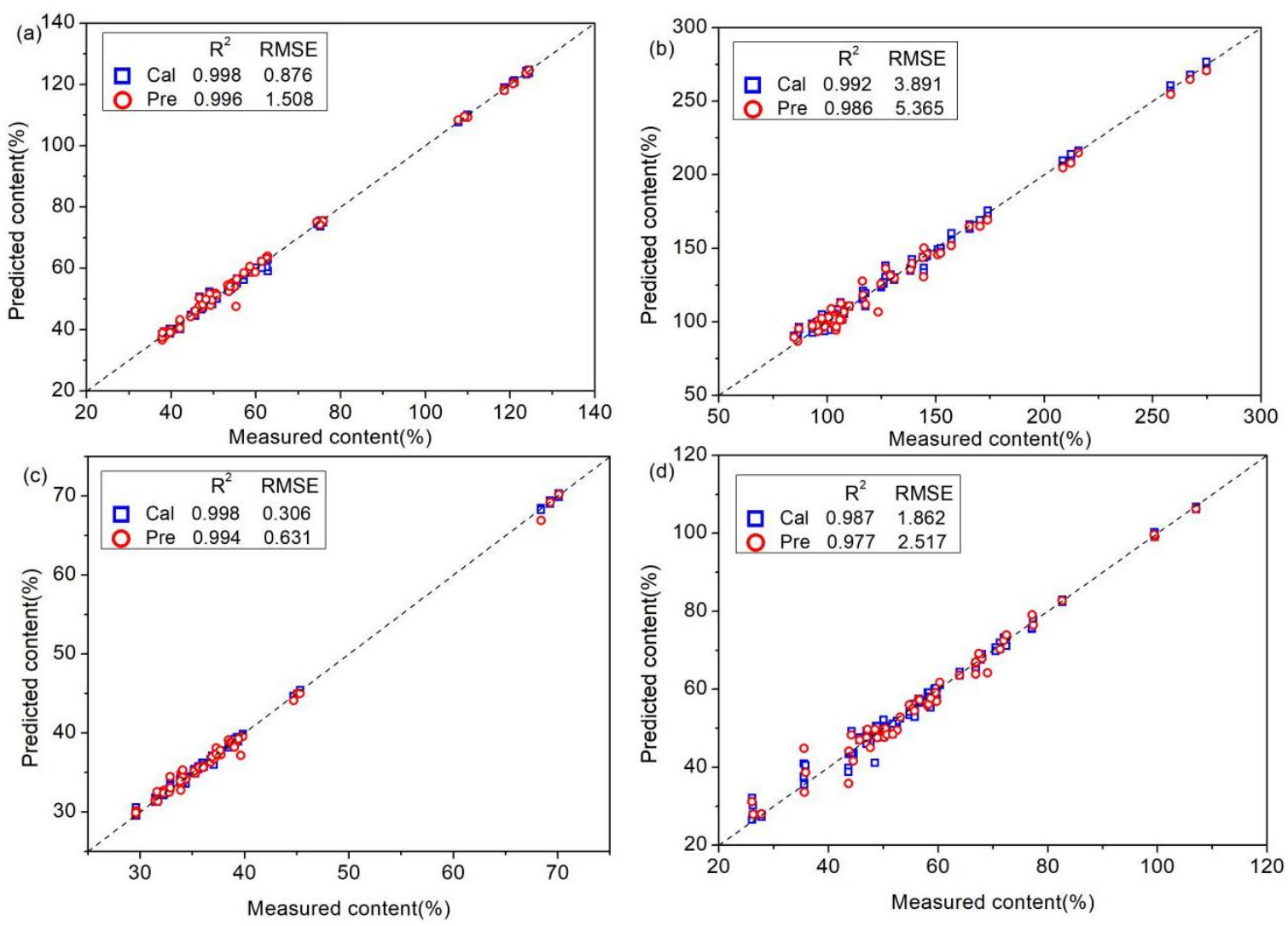

\section{Conclusions}

In this study, Vis-NIR spectroscopy technology was used to successfully predict the content spectral data. Based on different preprocessing methods, respective PLS prediction models were established, and the optimal preprocessing method was determined according to the performance of the models. For further analysis, CARS, SPA and RF were used to extract characteristic wavelengths from the preprocessed spectral data. And the calibration models of PLS, MLR, and full-wavelength model, the model based on the characteristic wavelength not only retains the 
accuracy of the model, but also reduces the number of input wavelengths and improves the detection efficiency. Among them, the LS-SVM model based on SPA is most suitable for detecting the content of tea polyphenols. In summary, CARS, SPA and RF are all very effective feature wavelength extraction methods. The results of this study show that the combination of Vis-NIR spectroscopy and chemometrics can effectively detect the content of tea polyphenols, and it has high scientific significance for rapid non-destructive testing of the physicochemical information of tea.

\section{Acknowledgement}

The authors are grateful for the National natural science foundation of China (41867020), Jiangxi provincial department of science and technology project.

\section{Funding}

This study was supported by grants from the National natural science foundation of China (41867020).

\section{Authors' contributions}

$\mathrm{HZ}$ and WD wrote original draft. WL, BZ and $\mathrm{HH}$ performed the experiments. $\mathrm{HC}, \mathrm{KM}$ and XL analyzed the data. All the authors discussed the results and collectively edited the manuscript. All authors read and approved the final manuscript. 


\title{
Availability of data and materials
}

The datasets during and/or analysed during the current study available from the corresponding author on reasonable request.

\section{Ethics approval and consent to participate}

Not applicable.

\section{Consent for publication}

\author{
Not applicable.
}

\section{Competing interests}

The authors declare that they have no competing interests.

\section{References}

1. Brahma N S, Sharmila S, Rakesh K S. Green tea catechin, epigallocatechin-3-gallate (EGCG): Mechanisms, perspectives and clinical applications. Biochemical Pharmacology. 2011;82(12).

2. Xinrong W, Yuefei W, Shikang Z, Ping X, Xianqiang Y. Research Progress on the Health Functions of Tea Polyphenols and Development of Healthy Foods. Journal of Tea Science. 2010;30(S1):501-505. 
357

358

359

360

361

362

363

364

365

366

367

368

369

370

371

372

3. HaiJun C. Study on the physiological health function of tea polyphenols and its detection methods. Light Industry Science and Technology. 2009;25(03):4-5.

4. Jinchuan Y, Xuemei B. Simultaneous Determination of Catechins and Caffeine Content in Tea by HPLC. Guizhou Agricultural Sciences. 2020;48(02):99-102.

5. Ainara G, Silvia F D V, Nerea E, Alberto D D, Juan M M. Non-destructive Spectroscopy combined with chemometrics as a tool for Green Chemical Analysis of environmental samples: A review. Trends in Analytical Chemistry. 2016;76.

6. Xiaolei Z, Wei X, Shanhu T. Rapid determination of moisture content in fresh tea leaves based on visible and near infrared spectroscopy. Journal of Southwest Minzu University(Natural Science Edition). 2018;44(04):352-357.

7. JuanJuan J. Detection of Heavy Metal Stress in Camellia sinensis and Physiologiacl Indices Changes Based on Spectral Technology. Zhejiang University. 2019

8. Dutta R, Stein A, Bhagat R M. Integrating satellite images and spectroscopy to measuring green and black tea quality. Food chemistry. 2011;127(2).

9. JinLei C, Ying S, De Z, DeJiang N, Pei L, Zhi Y. Application of surface enhanced Raman spectroscopy in tea safety inspection and quality analysis. Journal of Food Safety \& Quality. 2020;11(13):4304-4309.

10. Xiaoli B, Weihua G, Junhao K, Shunhang L, Changwen L. Establishment of a Method for the Rapid Measurement of Moisture, Caffeine and Tea-polyphenols in Instant Pu'er Tea by Near Infrared Spectroscopy. Science and Technology of Food Industry. 2019;40(01):234-238. 
377

378

11. Ze L, Hualin X, Lin C, Jianhua H. An Improved Weighted Partial Least Squares Method Coupled with Near Infrared Spectroscopy for Rapid Determination of Multiple Components and Anti-Oxidant Activity of Pu-Erh Tea. Molecules. 2018;23(5).

12. Yuying Z. Detection of the Tea Polyphenol in Green Tea Based on Spectroscopy. Zhejiang University. 2017

13. Yifeng H, Wentao D, Alireza S, Xiaoming W, Wei L, Baishao Z, Xuemei L, Ruili L, Hailiang Z, Xiaoli L. Development of simple identification models for four main catechins and caffeine in fresh green tea leaf based on visible and near-infrared spectroscopy. Computers and Electronics in Agriculture. 2020;173.

14. Zhang L, Sun H, Rao Z, Ji H. Non-destructive identification of slightly sprouted wheat kernels using hyperspectral data on both sides of wheat kernels. Biosystems Engineering. 2020;200.

15. Hongdong L, Yizeng L, Qingsong X, Dongsheng C. Key wavelengths screening using competitive adaptive reweighted sampling method for multivariate calibration. Analytica Chimica Acta. 2009;648(1).

16. Guo T, Jing H, Hong Y, Yuquan Z, Yanmei X, Shungeng M. Determination of active ingredients in matrine aqueous solutions by mid-infrared spectroscopy and competitive adaptive reweighted sampling. Optik - International Journal for Light and Electron Optics. 2016;127(3).

17. Mário C U A, Teresa C B S, Roberto K H G O, Takashi Y, Henrique C C, Valeria V. The successive projections algorithm for variable selection in spectroscopic multicomponent analysis. Chemometrics and Intelligent Laboratory Systems. 2001;57(2). 
397

18. Yujie W, Luqing L, Shanshan S, Ying L, Jingming N, Zhengzhu Z. Rapid detection of quality index of postharvest fresh tea leaves using hyperspectral imaging. Journal of the Science of Food and Agriculture. 2020;100(10).

19. Jingjing W, Muhammad Z, Peihuan H, Hao S, Quansheng C, Huanhuan L, Qin O, Zhiming G, Zhengzhu Z, Delian X. Evaluation of matcha tea quality index using portable NIR spectroscopy coupled with chemometric algorithms. Journal of the science of food and agriculture. 2019;99(11).

20. Mishra P, Nikzad L R. Partial least square regression versus domain invariant partial least square regression with application to near-infrared spectroscopy of fresh fruit. Infrared Physics \& Technology. 2020;111(prepublish).

21. Kumar K. Competitive adaptive reweighted sampling assisted partial least square analysis of excitation-emission matrix fluorescence spectroscopic data sets of certain polycyclic aromatic hydrocarbons. Spectrochimica acta. Part A, Molecular and biomolecular spectroscopy. 2020;244.

22. Hailiang Z, Shuai Z, Yin C, Wei L, Yifeng H, Dan T, Baishao Z, Xuemei L. Non-destructive determination of fat and moisture contents in Salmon ( Salmo salar ) fillets using near-infrared hyperspectral imaging coupled with spectral and textural features. Journal of Food Composition and Analysis. 2020;92.

23. Jiangbo L, Wenqian $\mathrm{H}$, Chunjiang Z, Baohua Z. A comparative study for the quantitative determination of soluble solids content, $\mathrm{pH}$ and firmness of pears by Vis/NIR spectroscopy. Journal of Food Engineering. 2013;116(2).

24. Yankun L, Xueguang S, Wensheng C. A consensus least squares support vector regression (LS-SVR) for analysis of near-infrared spectra of plant samples. Talanta. 2006;72(1). 
25. Xiaoli L, Chanjun S, Liubin L, Yong H. Determination of tea polyphenols content by infrared spectroscopy coupled with iPLS and random frog techniques. Computers and Electronics in Agriculture. 2015;112.

26. Komal G, James T E, Christopher J Z, Jeffrey J R. Tuning Excited State Isomerization Dynamics through Ground State Structural Changes in Analogous Ruthenium and Osmium Sulfoxide Complexes. Chemistry - A European Journal. 2013;19(35).

27. Xiaoli L, Juanjuan J, Chanjun S, Dapeng Y, Yufei L. Simultaneous determination of six main types of lipid-soluble pigments in green tea by visible and near-infrared spectroscopy. Food Chemistry. $2019 ; 270$.

28. Lee M, Hwang Y, Lee J, Choung M. The characterization of caffeine and nine individual catechins in the leaves of green tea (Camellia sinensis L.) by near-infrared reflectance spectroscopy. Food chemistry. 2014;158.

29. Li Y, Bin S, Jiancheng Y, Tianyu T, Yuan Y. Application of Different Smoothing Ensemble CARS Algorithm in Spectral Discrimination of Black Tea Grade. Spectroscopy and Spectral Analysis. 2020;40(10):3254-3259. 
Figures

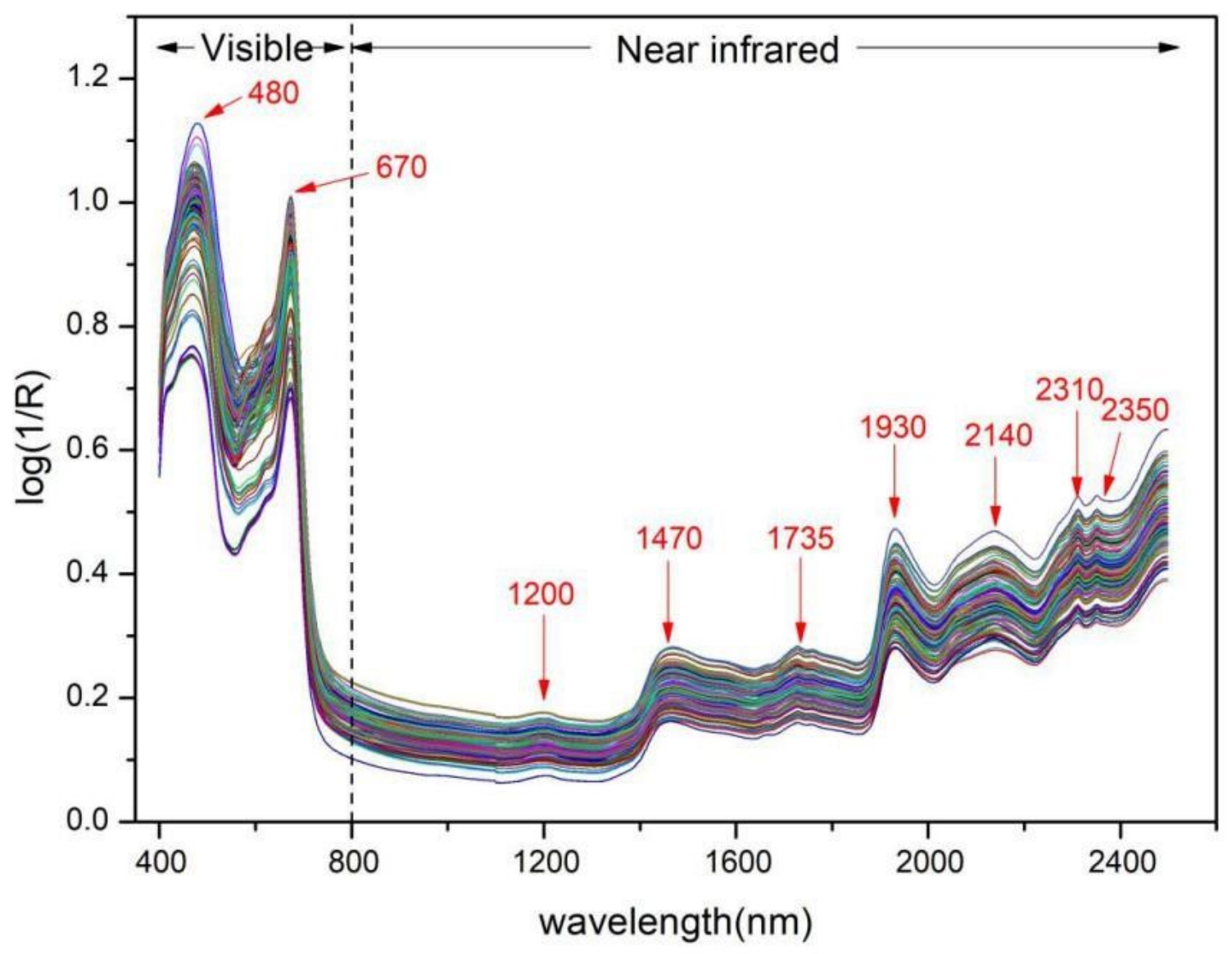

Figure 1

Visible and near-infrared spectroscopy of tea samples 

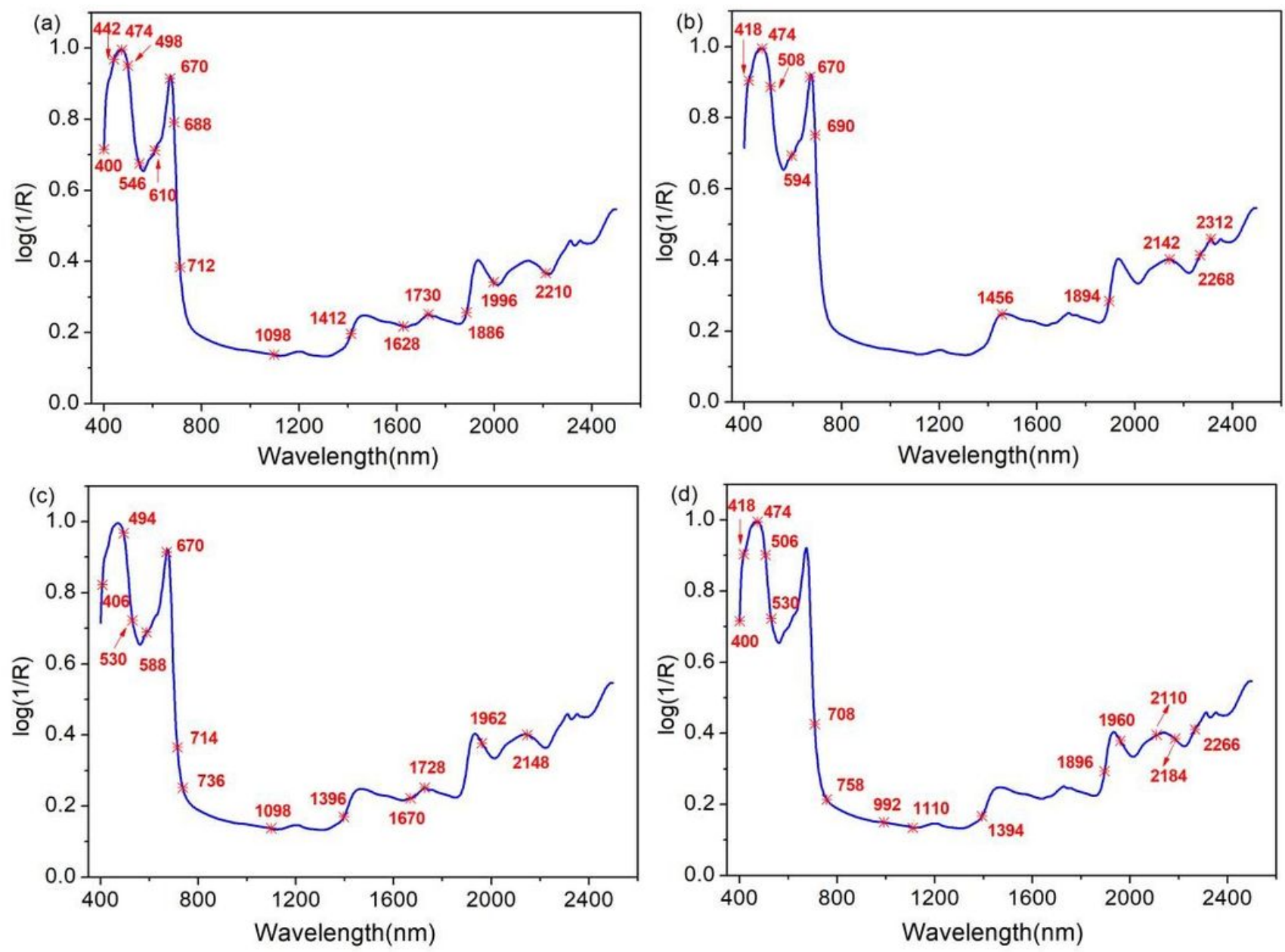

Figure 2

The characteristic wavelength is extracted from the optimal pretreatment spectrum based on SPA, (a)EGC (b)EGCG (c)EC (d)ECG 

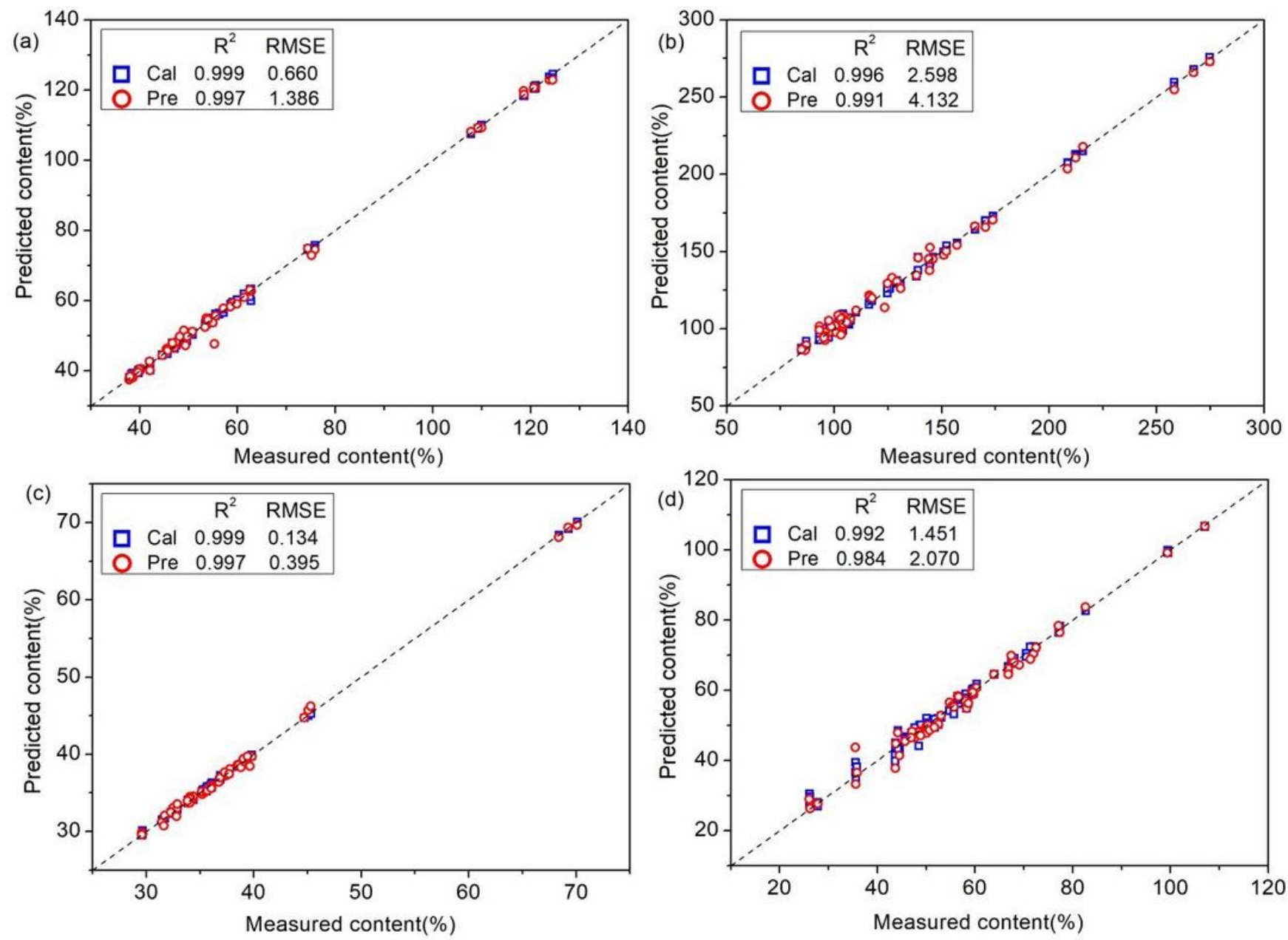

Figure 3

Scatter plot of four tea polyphenol LS-SVM models based on CARS, (a)EGC (b)EGCG (c)EC (d)ECG 

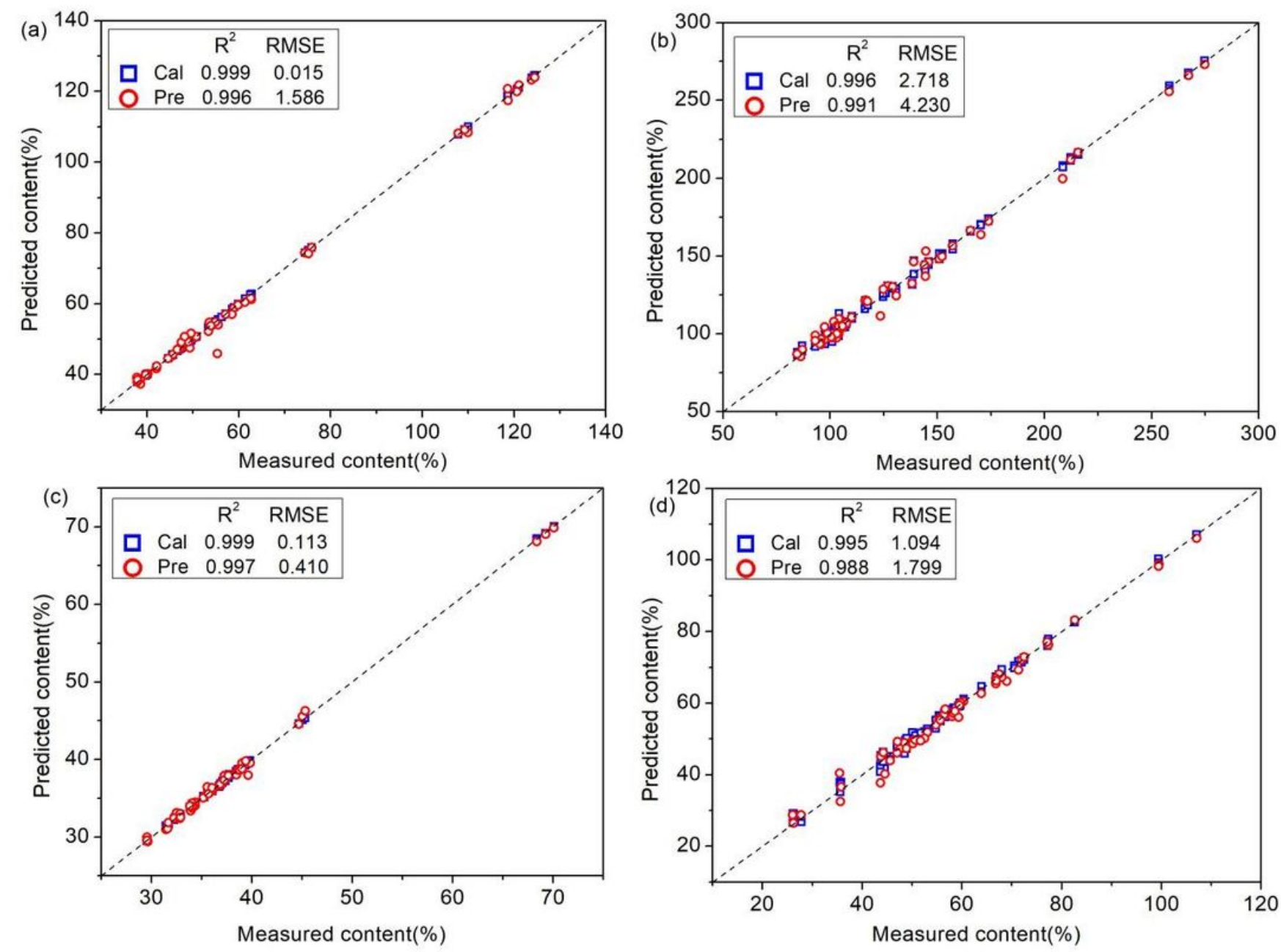

\section{Figure 4}

Scatter plot of four tea polyphenol LS-SVM models based on SPA, (a)EGC (b)EGCG (c)EC (d)ECG 

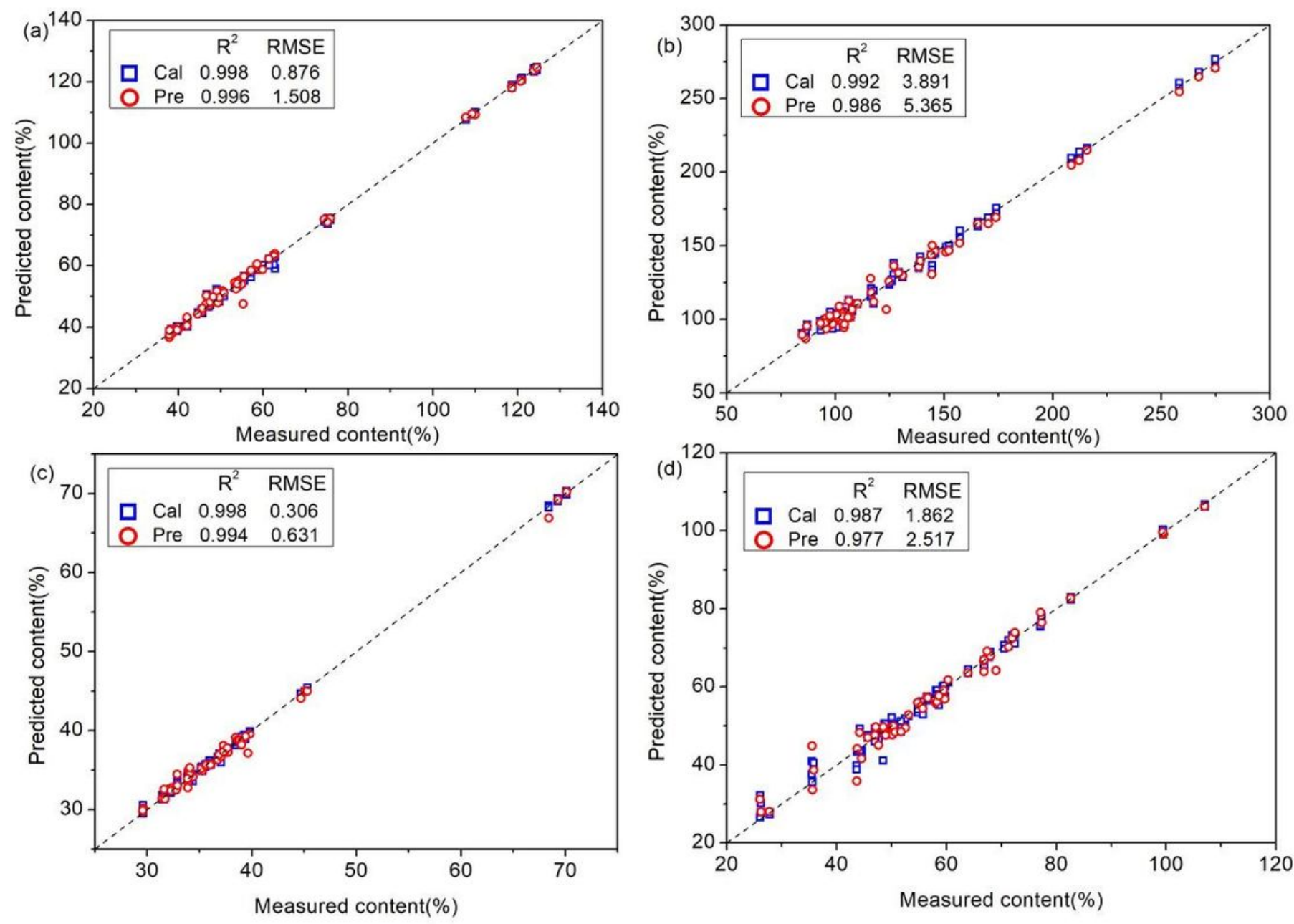

Figure 5

Scatter plot of four tea polyphenol LS-SVM models based on RF, (a)EGC (b)EGCG (c)EC (d)ECG 